\title{
Análise do conhecimento de Graduandos em Odontologia sobre o uso de anestésico local em pacientes com necessidades especiais
}

\author{
Knowledge Analysis of Dentistry Course Students about \\ local anaesthetics in especial patients
}

\author{
Romero Samarcos Mendes Pontanegra ${ }^{1}$ \\ Cecilia Corcino de Lucena Camboim ${ }^{1}$ \\ Julliana Cariry Palhano Freire ${ }^{2}$ \\ Marina Tavares Costa Nóbrega ${ }^{2}$ \\ JaQueLINE OLiveIRA BARRETO ${ }^{3}$ \\ Jalber Almeida dos Santos ${ }^{4}$ \\ EdUARDo DIAS-RIBEIRO
}

\begin{abstract}
RESUMO
Introdução: Os medicamentos utilizados por cirurgiões-dentistas auxiliam na prevenção e na cura de doenças bucais, bem como para tratamento em âmbito clínico, tornando necessário o conhecimento da interação do medicamento com a doença do paciente e a correta indicação para uso. Objetivo: Analisar o conhecimento de graduandos em Odontologia das Faculdades Integradas de Patos sobre o correto uso de anestésicos locais e vasoconstritores em pacientes portadores de asma, hipertireoidismo, diabetes, usuário de drogas antidepressivas, oxigenação deficiente e gestante. Material e Métodos: Foi realizado um estudo transversal com análise quantitativa, aplicando um questionário estruturado com nove perguntas aos alunos que concluíram a disciplina de anestesiologia. Os resultados foram tabulados no software IBM SPSS versão 20.0 e aplicou-se o teste Exato de Fisher. Resultados: A amostra foi composta por 81 alunos, sendo a maioria $\left(58 \%\right.$ ) do gênero feminino. Do total de alunos $44,4 \%$ pertenciam ao $7^{\circ}$ período e $92,6 \%$ atendiam na clínica escola. A maioria dos alunos não soube responder corretamente as perguntas realizadas, tendo um percentual que variou entre $16 \%$ e $69,1 \%$. Do total de perguntas, 8 tiveram um percentual de acerto inferior à $20 \%$. Uma das perguntas obteve acerto de 3,7\%. Apenas uma das questões teve um percentual de acertos favorável $(51,9 \%)$. Conclusões: Há uma deficiência no conhecimento dos alunos sobre o uso de anestésicos locais em pacientes portadores de necessidades especiais. A estimulação para obtenção desse conhecimento deve receber uma atenção maior, pois o uso de anestésicos locais está na prática diária dos cirurgiões-dentistas.
\end{abstract}

Palavras-chave: Anestésicos Locais; Vasoconstritores; Diabetes Mellitus; Antidepressivos Tricíclicos; Oxigenação.

\begin{abstract}
Introduction: The medications used by dentists help in preventing and curing oral diseases and for treatment in the clinical setting, requiring knowledge the interaction between the drug with the patient's condition and the correct indication for use. Aim: to analyze the knowledge of Dentistry course students of Patos Integrated College on the proper use of local anesthetics and vasoconstrictors in patients with asthma, hyperthyroidism, diabetes, antidepressant drugs user, poor oxygenation and pregnant. Material and Methods: A crosssectional study with a quantitative analysis was conducted by applying a questionnaire with nine questions to students who have completed the course of anesthesiology. The results were tabulated in IBM SPSS
\end{abstract}

Cirurgiões-Dentistas, Curso de Odontologia, Faculdades Integradas de Patos (FIP), Patos-PB.

Mestrandas em Ciências Odontológicas, Programa de Pós-graduação em Odontologia, Universidade Federal da Paraíba (UFPB), João Pessoa-PB.

3 Graduanda em Odontologia, Universidade Federal de Campina Grande (UFCG), campus de Patos-PB.

4 Professor Substituto do curso de Odontologia, Universidade Federal de Campina Grande (UFCG), campus de Patos-PB.

5 Professor Adjunto do curso de Odontologia, Universidade Federal de Campina Grande (UFCG), campus de Patos-PB. 
version 20.0 software and applied Fisher's exact test. Results: The sample consisted of 81 students, the majority $(58 \%)$ female. Of the students, $44.4 \%$ belonged to the 7 th period and $92.6 \%$ attended on the school clinic. Most students did not know how to correctly answer the questions asked, with a percentage ranging between $16 \%$ and $69.1 \%$. Of the total questions, 8 had a hit percentage lower than $20 \%$. One of the questions got $3.7 \%$ of correct answers. Only one of the questions had a favorable percentage of hit (51.9\%). Conclusions: There is a lack on students knowledge about the use of local anesthetics in patients with special needs. Stimulation for obtaining such knowledge must receive greater attention, since the use of local anesthetics is the daily practice of dentists.

Keywords: Local Anesthetics; Vasoconstrictor; Diabetes Mellitus; Tricyclic Antidepressive; Oxygenation.

\section{INTRODUÇÃO}

É um desafio para o cirurgião-dentista reverter o passado de sua profissão, a qual foi caricaturado pela sociedade como personagens de terror por muitas gerações, levando os indivíduos temer assistência de saúde bucal por medo de sentir dor e pelo o transtorno da ansiedade que desenvolveram com isso, embora menos, hoje essa problemática ainda existe..$^{1,2}$ Tendo em vista isso e que os medicamentos utilizados por cirurgiõesdentistas auxiliam na prevenção e na cura de doenças bucais, bem como para tratamento em âmbito clínico, para o profissional dessa área desassociar seus procedimentos clínicos com a percepção dolorosa o faz recorrer a crescente demanda de fármacos existentes que assegurem um conforto para o paciente durante a conduta clinica, promovendo dessa maneira, a necessidade da atenção constante com a terapêutica medicamentosa, principalmente dos anestésicos locais. ${ }^{3}$

A droga citada impede a sensação dolorosa bloqueando a condução nervosa de forma transitória sem perdas de consciência, sendo considerada no nível bom quando possui propriedades de baixa toxicidade, não irritabilidade aos tecidos, não lesione as estruturas nervosas, tenha início rápido e duração eficaz, além de ter menor competência alergênica e desta, a amida e o éster são os tipos de anestésicos mais utilizados na Odontologia, porém a primeira possui essas propriedades em maior potencial. ${ }^{2,4,5}$ Por isso, a escolha do sal anestésico para cada paciente é um passo importante para dar continuidade no procedimento planejado, o não conhecimento da interação do anestésico com casos mais específicos, como possíveis reações adversas e o cálculo da dosagem máxima permitida para cada paciente tratado, aumentam as chances de intercorrências durante o procedimento. ${ }^{1,4,6}$

Atualmente, os profissionais necessitam de uma precaução maior voltada para a saúde geral de cada tipo de paciente, sendo relevante $\mathrm{o}$ conhecimento teórico-prático sobre pacientes com necessidades especiais (PNE), termo o qual não se refere apenas a pacientes que tem deficiência física, motora e/ou psicológica, mas todos aqueles que fogem do padrão de normalidade, justificando a necessidade de uma abordagem especial momentânea ou indefinidamente para pacientes hipertensos, diabéticos, gestantes, asmáticos, com hipertireoidismo e diversos outros. $^{7}$ Conforme Mugayar ${ }^{8}$ quando é detectada alguma alteração do padrão de normalidade, o paciente se enquadra como $\mathrm{PNE}$, precisando o odontólogo fazer o planejamento do procedimento considerando essa informação para que seja tomada a escolha correta do anestésico. ${ }^{9}$

Devido à deficiência na literatura sobre o conhecimento por parte dos alunos, futuros cirurgiões-dentistas recém-formados, o objetivo desse estudo foi avaliar o conhecimento desses sobre o uso de anestésicos locais em PNE. Enfatiza-se a melhor indicação frente a diferentes tipos de necessidades especiais, a interação do anestésico com os medicamentos 
utilizados pelos pacientes, as indicações e contraindicações dos vasoconstritores. ${ }^{10}$

\section{Material e mÉtodos}

Tratou-se de um estudo observacional transversal com análise quantitativa. Aplicouse um questionário aos alunos da graduação em odontologia das Faculdades Integradas de Patos (FIP), Patos, Paraíba, Brasil, para obter o conhecimento dos mesmos sobre o uso de anestésicos locais em PNE (portadores de asma, hipertireoidismo, diabetes, usuário de drogas antidepressivas, oxigenação deficiente e gestante) ${ }^{5}$

Essa pesquisa foi aprovada pelo Comitê de Ética em Pesquisa das FIP sob número de parecer 1.262.831, respeitando-se a Resolução 466/12, do Conselho Nacional de Saúde. Foram incluídos na pesquisa, os alunos do curso de odontologia da instituição que aceitaram participar do estudo por meio de assinatura do Termo de Consentimento Livre e Esclarecido (TCLE) e que concluíram a disciplina de anestesiologia. Foram excluídos aqueles que se negaram a assinar o TCLE e aqueles que não quiseram responder ao questionário durante 0 contato com o pesquisador, resultando em uma amostra de 81 alunos.

A coleta de dados foi realizada por meio da aplicação de um questionário, utilizando um formulário estruturado com nove perguntas objetivas. As perguntas almejam obter dados do conhecimento dos graduandos sobre o uso de anestésico local em pacientes diabéticos, asmáticos, com hipertireoidismo, usuários de medicamentos antidepressivos, com oxigenação deficiente e gestante. Por se tratar de co-morbidades muito presentes atualmente no dia-a-dia do consultório odontológico. O questionário foi respondido na instituição de ensino escolhida para a pesquisa e tempo previsto para a duração das respostas foi em torno de 5 minutos.

As respostas foram tabuladas em uma planilha do software SPSS versão $20.0\left(\right.$ IBM $^{\circledR}$, Armonk, New York, USA) considerando um intervalo de confiança de $95 \%$, e aplicado o teste de exato de Fisher para determinar eventuais diferenças entre os alunos de acordo com o gênero, período cursado e se atender em clínica escola influencia no conhecimento gerado. Em seguida, as respostas do questionário foram dicotomizadas em certas e erradas, onde estão esclarecidas na discussão.

\section{Resultados}

De acordo com a Tabela 1, a qual mostra as frequências absolutas e percentuais das respostas dos questionários aplicados aos graduandos de odontologia das FIP, a maioria dos participantes pertenceu ao gênero feminino $(n=47 ; 58 \%)$, cursaram o sétimo período da grade curricular $(n=39 ; 44,4 \%)$ e atendiam pacientes na clínica escola $(n=75 ; 92,6 \%)$.

Umtotal de 56 alunos $(69,1 \%)$ respondeuque não sabia qual vasoconstritor é contraindicado para pacientes que fazem o uso de inibidores da monoamina oxidase (MAO). Quando questionados sobre o vasoconstritor que causa contração intrauterina e metemoglobinemia, 42 $(51,9 \%)$ dos alunos responderam felipressina. Além disso, quando perguntados sobre qual anestésico utilizariam como primeira escolha para pacientes asmáticos, mais de $50 \%$ dos alunos optaram em utilizar um anestésico sem vasoconstritor, $27(33,3 \%)$ responderam que utilizaria a Mepivacaína a $3 \%$ e 19 participantes $(23,5 \%)$ responderam que utilizaria a Lidocaína sem vasoconstritor. 
Tabela 1. Frequências absolutas e percentuais das respostas dos questionários aplicados aos graduandos em odontologia sobre o uso de anestésicos locais e vasoconstritores. ( $n=81$, Patos-PB).

\begin{tabular}{l|c|c}
\hline \multicolumn{1}{c|}{ Variáveis } & $\mathrm{n}$ & $\%$ \\
\hline Gênero & & \\
\hline Masculino & 34 & 42,0 \\
\hline Feminino & 47 & 58,0 \\
\hline Período & & \\
\hline $6^{\circ}$ & 27 & 55,6 \\
\hline $7^{\circ}$ & 39 & 44,4 \\
\hline $8^{\circ}$ & 15 & 18,5 \\
\hline Atende em clínica escola? & & \\
\hline Sim & 75 & 92,6 \\
\hline Não & 6 & 7,4 \\
\hline
\end{tabular}

Entre esses anestésicos, qual você utilizaria como primeira escolha para pacientes com asma?

\begin{tabular}{l|c|c}
\hline Articaína + epinefrina & 7 & 8,6 \\
\hline Prilocaína 3\% + Felipressina & 15 & 18,5 \\
\hline Mepivacaína 3\% & 27 & 33,3 \\
\hline Lidocaína sem vasoconstritor & 19 & 23,5 \\
\hline Não sei & 13 & 16,0 \\
\hline
\end{tabular}

Qual vasoconstritor contraindicado para pacientes usuários de inibidores da MAO?

\begin{tabular}{l|c|c}
\hline Fenilefrina & 3 & 3,7 \\
\hline Noradrenalina & 6 & 7,4 \\
\hline Levonordefrina & 2 & 2,5 \\
\hline Felipressina & 7 & 8,6 \\
\hline Adrenalina & 7 & 8,6 \\
\hline Não sei & 56 & 69,1 \\
\hline
\end{tabular}

Qual vasoconstritor causa a contração intra uterina e metemoglobinemia no feto e por isso é contra indicado em gestantes?

\begin{tabular}{l|c|c}
\hline Adrenalina & 9 & 11,1 \\
\hline Fenilefrina & 5 & 6,2 \\
\hline Felipressina & 42 & 51,9 \\
\hline Noraepinefrina & 5 & 6,2 \\
\hline Epinefrina & 9 & 11,1 \\
\hline Não sei & 11 & 13,6 \\
\hline Total & $\mathbf{8 1}$ & $\mathbf{1 0 0 \%}$ \\
\hline
\end{tabular}

A Tabela 2 apresenta a distribuição dos alunos participantes da pesquisa de acordo com o gênero e as respostas dos questionários sobre o uso de anestésicos e vasoconstritores. $\mathrm{O}$ teste exato de Fisher revelou diferença estatisticamente significativa entre o gênero dos alunos e o conhecimento sobre a indicação correta do anestésico local de primeira escolha para pacientes com asma $(p=0,004)$. Entre os alunos do gênero masculino, 8 (23,5\%) demonstraram ter conhecimento adequado, 23 $(67,6 \%)$ demonstraram não ter o conhecimento adequado e $3(8,8 \%)$ não souberam responder sobre qual anestésico utilizar em pacientes com deficiência na oxigenação, ao passo que entre os alunos do gênero feminino, 2 (4,3\%) demonstraram ter o conhecimento, 31 (66\%) demonstraram não ter o conhecimento e 14 $(29,8 \%)$ não souberam responder.

A Tabela 3 apresenta a distribuição dos alunos participantes da pesquisa de acordo com o período que está cursando e as respostas dos questionários sobre o uso de anestésicos e vasoconstritores. Quando questionados sobre qual anestésico local utilizaria em pacientes usuários de drogas antidepressivas, aplicando o teste exato de Fisher observamos uma estatística altamente significativa $(p=0,005)$, no $6^{\circ}$ período: $0(0 \%)$ acertaram a resposta, no $7^{\circ}$ período apenas $8(20,5 \%)$ e no $8^{\circ}$ período 6 alunos $(40 \%)$ responderam corretamente. O teste também revelou uma diferença estatisticamente significativa entre os períodos dos alunos e o conhecimento sobre qual anestésico deve ser evitado em pacientes com deficiência na oxigenação $(p=0,03), 6^{\circ}$ período $0 \%$ mostrou ter conhecimento sobre o assunto, $74,1 \%$ erraram a questão e $25,9 \%$ não souberam responder, $7^{\circ}$ período $15,4 \%$ mostraram ter conhecimento, $71,8 \%$ erraram a questão e $12,8 \%$ não souberam responder e $08^{\circ}$ período teve $26,7 \%$ de alunos que mostraram ter conhecimento sobre o assunto, $40 \%$ erraram a questão e $33,3 \%$ não souberam responder. 
Tabela 2. Distribuição dos alunos participantes da pesquisa de acordo com o gênero e as respostas dos questionários. $(n=81$, Patos-PB).

\begin{tabular}{|c|c|c|c|c|c|c|c|}
\hline \multirow{3}{*}{ Variáveis } & \multicolumn{4}{|c|}{ Gênero } & & & \multirow{3}{*}{ p-valor } \\
\hline & \multicolumn{2}{|c|}{ Masculino } & \multicolumn{2}{|c|}{ Feminino } & \multicolumn{2}{|c|}{ Total } & \\
\hline & $\mathbf{n}$ & $\%$ & $\mathbf{n}$ & $\%$ & $\mathbf{n}$ & $\%$ & \\
\hline \multicolumn{8}{|c|}{ Entre esses anestésicos, qual você utilizaria como primeira escolha para pacientes com asma? } \\
\hline Certo & 4 & 11,8 & 11 & 23,4 & 15 & 18,5 & \multirow[t]{3}{*}{0,004} \\
\hline Errado & 29 & 85,3 & 24 & 51,1 & 53 & 65,4 & \\
\hline Não Sabe & 1 & 2,9 & 12 & 25,5 & 13 & 16,0 & \\
\hline
\end{tabular}

Entre esses anestésicos locais, qual você utilizaria como primeira escolha em pacientes com hipertireoidismo?

\begin{tabular}{l|c|c|c|c|c|c|c|}
\hline Certo & 8 & 23,5 & 8 & 17,0 & 16 & 19,8 & 0,1 \\
\hline Errado & 19 & 55,9 & 20 & 42,6 & 39 & 48,1 \\
\hline Não Sabe & 7 & 20,6 & 19 & 40,4 & 26 & 32,1 & \\
\hline
\end{tabular}

Qual anestésico mais indicado para pacientes diabético insulino dependente?

\begin{tabular}{l|c|c|c|c|c|c|c|}
\hline Certo & 5 & 14,7 & 7 & 14,9 & 12 & 14,8 & 0,3 \\
\hline Errado & 24 & 70,6 & 27 & 57,4 & 51 & 63,0 \\
\hline Não Sabe & 5 & 14,7 & 13 & 27,7 & 18 & 22,2 & \\
\hline
\end{tabular}

Paciente usuário de drogas antidepressivas, qual anestésico você indicaria?

\begin{tabular}{l|c|c|c|c|c|c|c|}
\hline Certo & 9 & 26,5 & 5 & 10,6 & 14 & 17,3 & 0,1 \\
\hline Errado & 15 & 44,1 & 26 & 55,3 & 41 & 50,6 \\
\hline Não Sabe & 10 & 29,4 & 16 & 34,0 & 26 & 32,1 & \\
\hline
\end{tabular}

Qual vasoconstritor deve ser mais evitado em pacientes usuários de antidepressivos tricíclicos?

\begin{tabular}{l|c|c|c|c|c|c|c|}
\hline Certo & 4 & 11,8 & 2 & 4,3 & 6 & 7,4 & 0,2 \\
\hline Errado & 14 & 41,2 & 26 & 55,3 & 40 & 49,4 \\
\hline Não Sabe & 16 & 47,1 & 19 & 40,4 & 35 & 43,2 & \\
\hline
\end{tabular}

O uso de adrenalina em diabéticos descompensados pode causar:

\begin{tabular}{l|c|c|c|c|c|c|c|c|}
\hline Certo & 3 & 8,8 & 1 & 2,1 & 4 & 4,9 & 0,3 \\
\hline Errado & 22 & 64,7 & 30 & 63,8 & 52 & 64,2 \\
\hline Não Sabe & 9 & 26,5 & 16 & 34,0 & 25 & 30,9 & \\
\hline O vasoconstritor contraindicado em pacientes usuários de inibidores da MAO é? & 2 & 5,9 & 1 & 2,1 & 3 & 3,7 & \multirow{2}{*}{0,6} \\
\hline Certo & 2 & 10 & 29,4 & 13 & 27,7 & 23 & 28,4 \\
\hline Errado & 22 & 64,7 & 33 & 70,2 & 55 & 67,9 & \\
\hline Não Sabe & 22 &
\end{tabular}

Paciente com oxigenação deficiente, qual anestésico local deve ser evitado?

\begin{tabular}{l|c|c|c|c|c|c|c|}
\hline Certo & 8 & 23,5 & 2 & 4,3 & 10 & 12,3 & 0,06 \\
\hline Errado & 23 & 67,6 & 31 & 66,0 & 54 & 66,7 \\
\hline Não Sabe & 3 & 8,8 & 14 & 29,8 & 17 & 21,0 & \\
\hline
\end{tabular}

Qual vasoconstritor causa a contração intra uterina e metemoglobinemia no feto e por isso é contra indicado em gestantes?

\begin{tabular}{l|c|c|c|c|c|c|c|}
\hline Certo & 18 & 52,9 & 23 & 48,9 & 41 & 50,6 & 0,9 \\
\hline Errado & 12 & 35,3 & 17 & 36,2 & 29 & 35,8 \\
\hline Não Sabe & 4 & 11,8 & 7 & 14,9 & 11 & 13,6 & \\
\hline
\end{tabular}


Tabela 3. Distribuição dos alunos participantes da pesquisa de acordo com o período semestral e as respostas dos questionários. ( $n=81$, Patos-PB).

\begin{tabular}{|c|c|c|c|c|c|c|c|c|c|c|}
\hline \multirow{3}{*}{ Variáveis } & \multicolumn{6}{|c|}{ Período Semestral } & \multirow{2}{*}{\multicolumn{3}{|c|}{ Total }} & \multirow{3}{*}{ p-valor } \\
\hline & \multicolumn{2}{|c|}{$6^{\circ}$} & \multicolumn{2}{|c|}{$7^{\circ}$} & \multicolumn{2}{|c|}{$8^{\circ}$} & & & & \\
\hline & $\mathbf{n}$ & $\%$ & $\mathbf{n}$ & $\%$ & $\mathbf{n}$ & $\%$ & $\mathbf{n}$ & $\%$ & & \\
\hline \multicolumn{10}{|c|}{ Entre esses anestésicos, qual você utilizaria como primeira escolha para pacientes com asma? } & \\
\hline Certo & 3 & 11,1 & 7 & 17,9 & 5 & 33,3 & 15 & 18,5 & \multirow{3}{*}{0,07} & \\
\hline Errado & 16 & 59,3 & 29 & 74,4 & 8 & 53,3 & 53 & 65,4 & & \\
\hline Não Sabe & 8 & 29,6 & 3 & 7,7 & 2 & 13,3 & 13 & 16,0 & & \\
\hline \multicolumn{10}{|c|}{$\begin{array}{l}\text { Entre esses anestésicos locais, qual você utilizaria como primeira escolha em pacientes com } \\
\text { hipertireoidismo? }\end{array}$} & \\
\hline Certo & 5 & 18,5 & 7 & 17,9 & 4 & 26,7 & 16 & 19,8 & \multirow{3}{*}{0,9} & \\
\hline Errado & 13 & 48,1 & 20 & 51,3 & 6 & 40,0 & 39 & 48,1 & & \\
\hline Não Sabe & 9 & 33,3 & 12 & 30,8 & 5 & 33,3 & 26 & 32,1 & & \\
\hline \multicolumn{10}{|c|}{ Qual anestésico mais indicado para pacientes diabético insulino dependente? } & \\
\hline Certo & 4 & 14,8 & 7 & 17,9 & 1 & 6,7 & 12 & 14,8 & \multirow{3}{*}{0,3} & \\
\hline Errado & 14 & 51,9 & 26 & 66,7 & 11 & 73,3 & 51 & 63,0 & & \\
\hline Não Sabe & 9 & 33,3 & 6 & 15,4 & 3 & 20,0 & 18 & 22,2 & & \\
\hline
\end{tabular}

Paciente usuário de drogas antidepressivas, qual anestésico você indicaria?

\begin{tabular}{|c|c|c|c|c|c|c|c|c|c|}
\hline Certo & 0 & 0,0 & 8 & 20,5 & 6 & 40,0 & 14 & 17,3 & \multirow{3}{*}{0,005} \\
\hline Errado & 13 & 48,1 & 21 & 53,8 & 7 & 46,7 & 41 & 50,6 & \\
\hline Não Sabe & 14 & 51,9 & 10 & 25,6 & 2 & 13,3 & 26 & 32,1 & \\
\hline \multicolumn{10}{|c|}{ Qual vasoconstritor deve ser mais evitado em pacientes usuários de antidepressivos tricíclicos? } \\
\hline Certo & 3 & 11,1 & 2 & 5,1 & 1 & 6,7 & 6 & 7,4 & \multirow{3}{*}{0,1} \\
\hline Errado & 8 & 29,6 & 24 & 61,5 & 8 & 53,3 & 40 & 49,4 & \\
\hline Não Sabe & 16 & 59,3 & 13 & 33,3 & 6 & 40,0 & 35 & 43,2 & \\
\hline
\end{tabular}

O uso de adrenalina em diabéticos descompensados pode causar:

\begin{tabular}{|c|c|c|c|c|c|c|c|c|c|}
\hline Certo & 2 & 7,4 & 1 & 2,6 & 1 & 6,7 & 4 & 4,9 & \multirow{3}{*}{0,3} \\
\hline Errado & 16 & 59,3 & 29 & 74,4 & 7 & 46,7 & 52 & 64,2 & \\
\hline Não Sabe & 9 & 33,3 & 9 & 23,1 & 7 & 46,7 & 25 & 30,9 & \\
\hline
\end{tabular}

O vasoconstritor contraindicado em pacientes usuários de inibidores da MAO é?

\begin{tabular}{l|c|c|c|c|c|c|c|c|}
\hline Certo & 0 & 0,0 & 3 & 7,7 & 0 & 0,0 & 3 & 3,7 \\
\hline Errado & 7 & 25,9 & 15 & 38,5 & 1 & 6,7 & 23 & 28,4 \\
\hline Não Sabe & 20 & 74,1 & 21 & 53,8 & 14 & 93,3 & 55 & 67,9 \\
\hline
\end{tabular}

Paciente com oxigenação deficiente, qual anestésico local deve ser evitado?

\begin{tabular}{l|c|c|c|c|c|c|c|c}
\hline Certo & 0 & 0,0 & 6 & 15,4 & 4 & 26,7 & 10 & 12,3 \\
\hline Errado & 20 & 74,1 & 28 & 71,8 & 6 & 40,0 & 54 & 66,7 \\
\hline Não Sabe & 7 & 25,9 & 5 & 12,8 & 5 & 33,3 & 17 & 21,0 \\
\hline
\end{tabular}

Qual vasoconstritor causa a contração intra uterina e metemoglobinemia no feto e por isso é contra indicado em gestantes?

\begin{tabular}{|c|c|c|c|c|c|c|c|c|c|}
\hline Certo & 13 & 48,1 & 20 & 51,3 & 8 & 53,3 & 41 & 50,6 & \multirow{3}{*}{0,2} \\
\hline Errado & 8 & 29,6 & 17 & 43,6 & 4 & 26,7 & 29 & 35,8 & \\
\hline Não Sabe & 6 & 22,2 & 2 & 5,1 & 3 & 20,0 & 11 & 13,6 & \\
\hline
\end{tabular}


A Tabela 4 (na página seguinte) apresenta a distribuição dos alunos participantes da pesquisa de acordo com a atuação em clínica escola ou não, e as respostas dos questionários sobre o uso de anestésicos e vasoconstritores em PNE. Com base nisso o teste exato de Fisher mostrou significância estatística quando questionados sobre qual vasoconstritor causa contração intrauterina e metemoglobinemia no feto e por isso é contra indicado o uso em gestantes $(p=0,02)$, dos alunos que atendem em clínica escola 53,3\% acertaram sobre a contra-indicação, 36\% erraram e 10,7\% não souberam responder, já os alunos que não atendem em clínica escola 16,7\% acertaram sobre a contra indicação, 33,3\% erraram e $50 \%$ não souberam responder. Os alunos de ambos os grupos mostraram pouco conhecimento em relação ao anestésico mais indicado para pacientes diabético insulino dependente, dos que atendem em clínica escola $16 \%$ mostraram ter conhecimento, $62,7 \%$ mostraram não ter conhecimento e $21,3 \%$ não souberam responder, no grupo dos alunos que não atendem na clínica escola $0 \%$ mostrou ter conhecimento, 66,7\% mostrou não ter conhecimento e 33,3\% não souberam responder à pergunta.

\section{Dıscussão}

A descoberta de fármacos que causam perda da sensibilidade foi um marco para a Odontologia, pois proporcionou a realização de procedimentos cirúrgicos sem dor, fato que até então era considerado uma utopia nos meios acadêmicos. Sabe-se que a solução anestésica deve ser empregada de acordo com a história médica-dentária do paciente e do procedimento a ser realizado. Por isso, uma boa anamnese evita complicações sistêmicas advindas desses sais e possibilita segurança no atendimento. ${ }^{11-13}$
Por meio dos 81 questionários avaliados nesse estudo, quando foram comparados os resultados por período $\left(6^{\circ}, 7^{\circ} \mathrm{e} 8^{\circ}\right)$, percebeuse um melhor desempenho dos participantes dos períodos mais avançados do curso, mesmo sendo uma minoria que mostrou compreender o tema, o que pode ser explicado pelo o tempo de experiência em clínica e maior pratica atendendo vários tipos de pacientes os fez ter maior aprendizado, embora ainda apresentem uma insegurança por parte da correta indicação do anestésico local quando comparados com pacientes saudáveis, o que se assemelha com o estudo de Vasconcelos et al. ${ }^{14}$ Todavia, diferem os estudos de Guzmán Alvarez, Campos Sepúlveda, Matínez González ${ }^{15}$ e Caneppele et al. ${ }^{1}$, os quais verificaram que o maior tempo de experiência profissional e clínica para cirurgiões-dentistas e discentes respectivamente, não está associado em maior entendimento sobre a temática abordada.

Esses resultados insatisfatórios podem estar relacionados também a falta de associação da vivência clínica com situações reais e práticas em pré-clínicas dos cursos de farmacologia e terapêutica frente a pacientes que venham necessitar de algum tipo de necessidade especial ou até mesmo a falta do módulo teórico desse conteúdo dentro dessas disciplinas. ${ }^{15}$

Em relação ao que se utilizaria como primeira escolha para diferentes tipos de necessidades dos pacientes, os graduandos responderam que usariam a Mepivacaína, o que é confirmado por Veering ${ }^{16}$, porém se discorda com o feito por Vanconcellos et al. ${ }^{14}$, onde a minoria respondeu acertadamente a Prilocaína + Felipressina como anestésico de primeira escolha para os determinados grupos de PNE, embora também assemelhe-se com esses no que diz respeito com a insuficiência do conhecimento sobre anestésicos em pacientes com doenças sistêmicas. 
Tabela 4. Distribuição dos alunos participantes da pesquisa de acordo com atendimento em clínica escola e as respostas dos questionários sobre uso de anestésicos locais em PNE. (n=81, Patos-PB).

\begin{tabular}{l|c|c|c|c|c|c|c}
\hline & \multicolumn{3}{|c|}{ Atende em clínica escola? } & & \\
\hline \multirow{2}{*}{ Variáveis } & \multicolumn{2}{|c|}{ Sim } & \multicolumn{2}{|c|}{ Não } & \multicolumn{2}{|c|}{ Total } & p-valor \\
\cline { 2 - 7 } & $\mathbf{n}$ & $\%$ & $\mathbf{n}$ & $\%$ & $\mathbf{n}$ & $\%$ & \\
\hline
\end{tabular}

Entre esses anestésicos, qual você utilizaria como primeira escolha para pacientes com asma?

\begin{tabular}{l|c|c|c|c|c|c|}
\hline Certo & 15 & 20,0 & 0 & 0,0 & 15 & 18,5 \\
\hline Errado & 49 & 65,3 & 4 & 66,7 & 53 & 65,4 \\
\hline Não Sabe & 11 & 14,7 & 2 & 33,3 & 13 & 16,0 \\
\hline
\end{tabular}

Entre esses anestésicos locais, qual você utilizaria como primeira escolha em pacientes com hipertireoidismo?

\begin{tabular}{l|c|c|c|c|c|c|c|}
\hline Certo & 16 & 21,3 & 0 & 0,0 & 16 & 19,8 & 0,1 \\
\hline Errado & 37 & 49,3 & 2 & 33,3 & 39 & 48,1 & 0,1 \\
\hline Não Sabe & 22 & 29,3 & 4 & 66,7 & 26 & 32,1 & \\
\hline
\end{tabular}

Qual anestésico mais indicado para pacientes diabético insulino dependente?

\begin{tabular}{l|c|c|c|c|c|c|c|}
\hline Certo & 12 & 16.0 & 0 & 0,0 & 12 & 14,8 & \\
\hline Errado & 47 & 62,7 & 4 & 66,7 & 51 & 63,0 & 0,5 \\
\hline Não Sabe & 16 & 21,3 & 2 & 33,3 & 18 & 22,2 & \\
\hline
\end{tabular}

Paciente usuário de drogas antidepressivas, qual anestésico você indicaria?

\begin{tabular}{l|c|c|c|c|c|c|c|}
\hline Certo & 14 & 18,7 & 0 & 0,0 & 14 & 17,3 & \\
\hline Errado & 39 & 52,0 & 2 & 33,3 & 41 & 50,6 & 0,1 \\
\hline Não Sabe & 22 & 29,3 & 4 & 66,7 & 26 & 32,1 & \\
\hline
\end{tabular}

Qual vasoconstritor deve ser mais evitado em pacientes usuários de antidepressivos tricíclicos?

\begin{tabular}{|c|c|c|c|c|c|c|c|}
\hline Certo & 6 & 8,0 & 0 & 0,0 & 6 & 7,4 & \multirow{3}{*}{0,1} \\
\hline Errado & 39 & 52,0 & 1 & 16,7 & 40 & 49,4 & \\
\hline Não Sabe & 30 & 30,0 & 5 & 83,3 & 35 & 43,2 & \\
\hline \multicolumn{8}{|c|}{ O uso de adrenalina em diabéticos descompensados pode causar: } \\
\hline Certo & 3 & 4,0 & 1 & 16,7 & 4 & 4,9 & \multirow{3}{*}{0,3} \\
\hline Errado & 49 & 65,3 & 3 & 50,0 & 52 & 64,2 & \\
\hline Não Sabe & 23 & 30,7 & 2 & 33,3 & 25 & 30,9 & \\
\hline \multicolumn{8}{|c|}{ O vasoconstritor contraindicado em pacientes usuários de inibidores da MAO é? } \\
\hline Certo & 3 & 4,0 & 0 & 0,0 & 3 & 3,7 & \multirow{3}{*}{0,6} \\
\hline Errado & 22 & 29,3 & 1 & 16,7 & 23 & 28,4 & \\
\hline Não Sabe & 50 & 66,7 & 5 & 83,3 & 55 & 67,9 & \\
\hline \multicolumn{8}{|c|}{ Paciente com oxigenação deficiente, qual anestésico local deve ser evitado? } \\
\hline Certo & 10 & 13,3 & 0 & 0,0 & 10 & 12,3 & \multirow{3}{*}{0,1} \\
\hline Errado & 51 & 68,0 & 3 & 50,0 & 54 & 66,7 & \\
\hline Não Sabe & 14 & 18,7 & 3 & 50,0 & 17 & 21,0 & \\
\hline \multicolumn{8}{|c|}{$\begin{array}{l}\text { Qual vasoconstritor causa a contração intra uterina e metemoglobinemia no feto e por isso é contra indicado } \\
\text { em gestantes? }\end{array}$} \\
\hline Certo & 40 & 53,3 & 1 & 16,7 & 41 & 50,6 & \multirow{3}{*}{0,02} \\
\hline Errado & 27 & 36,0 & 2 & 33,3 & 19 & 35,8 & \\
\hline Não Sabe & 8 & 10,7 & 3 & 50,0 & 11 & 13,6 & \\
\hline
\end{tabular}


No presente estudo ainda foi visto que a maior parte da amostra entrevistada $(69,1 \%)$ respondeu que não sabia qual vasoconstritor é contraindicado para pacientes que fazem o uso de inibidores da MAO, dado preocupante, pois segundo Chioca et al. ${ }^{9}$ e Vanconcellos et al. ${ }^{14}$, essa medicação quando associados com substâncias simpatomiméticas, assim como drogas antidepressivas, quadros de hipertireoidismo e diabetes melitus podem causar um pico hipertensivo durante 0 procedimento.

Uma informação relevante para a prática clínica e o futuro profissional dos discentes é que se recomendam anestésicos locais associados com adrenalina para pacientes insulinodependente e para asmáticos, visto que para esses últimos pode até ter ação benéfica por ação sobre os receptores $\beta 2$ (broncodilatação), de toda forma deve-se ter atenção, pois na verdade eles se enquadram no grupo de risco a pacientes alérgicos a anestesia local. Diferente dos que sofrem de hipertireoidismo, sendo nesse caso o vasoconstritor mais recomendado a felipressina, com exceção de pacientes grávidas. ${ }^{11,17,18}$

A metemoglobinemia é uma doença sanguínea congênita ou adquirida caracterizada pelo nível aumentado de meta-hemoglobina que é uma forma de hemoglobina que está no estado de $\mathrm{Fe}^{3+}$ e não em $\mathrm{Fe}^{2+}$ como a hemoglobina normal, e não se liga ao oxigênio, diferentemente da oxi-hemoglobina. Por sua vez a Prilocaína através da ortotoluidina que é o seu principal metabólico causa uma oxidação na hemoglobina provocando cianose, depressão respiratória, cansaço, letargia e dificuldade respiratória. ${ }^{11,12,17}$

Uma sugestão prudente é que se aumente a carga-horária das disciplinas de Farmacologia e Terapêutica para que os alunos consigam ao menos simular situações em que irão atender pacientes com alguma necessidade especial, e assim façam administração da anestesia e do vasoconstrictor de modo eficaz, além da atenção com anamnese e cuidados clínicos, evitando eventuais riscos de reações e/ou intercorrências. ${ }^{12,19}$

\section{Conclusão}

A maioria dos entrevistados mostraram um conhecimento abaixo do esperado relacionado à indicação correta de um anestésico local para pacientes com as co-morbidades: asma, hipertireoidismo, diabetes, usuário de drogas antidepressivas, oxigenação deficiente e gestante. O conhecimento adequado sobre indicações, contraindicações e vasoconstrictores foi insuficiente, bem como o mecanismo que ocorre quando aplicado um anestésico incorretamente nesses grupos de pessoas.

\section{REFERÊNCIAS}

1. Caneppele TMF, Yamamoto EC, Souza AC, Valera MC, Araújo MAM. Conhecimento dos cirurgiões-dentistas sobre o atendimento de pacientes especiais: hipertensos, diabéticos e gestantes. J Biodent and Biomat. 2011; (1): 31-41.

2. Ferraz GR, Nascimento TMT, Menezes-Filho PF, Fernandes RSM. Avaliação do grau de ansiedade dos pacientes no pré-cirúrgico de Implantodontia. Full Dent Sci. 2014; 5(18): 258-62.

3. Carvalho JCA. Farmacologia dos anestésicos locais. Rev Bras Anestesiol. 1994; 44(1): 7582.

4. Catterall W, Mackie K. Anestésicos locais. In: Gilman AG, Hardman JG, Limbird LE. As bases farmacológicas da terapêutica. $10^{\mathrm{a}}$ ed. Rio de Janeiro: McGraw-Hill; 2003. 279-92.

5. Malamed SF. Manual de anestesia local. $6^{\text {a }}$ ed. Rio de Janeiro: Elsevier; 2013. 292-340.

6. Anjos ED, Carvalho RWF. Complicações sistêmicas em anestesia local. In: Lubiana NB. Pro-Odonto Cirurgia. $2^{\text {a }}$ Ed. Porto Alegre: Artmed; 2007. 143-78.

7. Vieira GF, Gonçalves EAN, Agra CM. Anestesia odontológica: segurança e sucesso - parte 1.

Rev Assoc Paul Cirur Dent. 2000; 54(1): 42-5. 
8. Mugayar LRF. Pacientes portadores de necessidades especiais: manual de odontologia e saúde oral. São Paulo: Pancast; 2000. 262.

9. Chioca LR, Segura RCF, Andreatini R, Losso EM. Antidepressivos e anestésicos locais: interações medicamentosas de interesse odontológico. Rev Sul-Bras Odontol. 2010; 7(4): 466-73.

10. Volpato MC, Ranali J. Reações à superdosagem das soluções anestésicas locais In: Andrade ED, Ranali J. Emergências médicas em odontologia. $3^{a}$ ed. São Paulo: Artes Médicas; 2011. 131-6.

11. Wannmacher L. Anestésicos locais. In: Wannmacher L, Ferreira MBC. Farmacologia clínica para dentistas. Rio de Janeiro: Guanabara Koogan; 1995. 74-9, 104-16.

12. Tenis CA. Avaliação do conhecimento científico dos alunos quarto e quinto anos do curso de odontologia da Unisa, quanto ao uso clínico dos anestésicos locais e as indicações em pacientes com distúrbios sistêmicos. (Dissertação) FO/USP. São Paulo, 2001. 124 p.

13. Carvalho RWF, Pereira CU, Anjos ED, Laureano Filho JR, Vasconcelos BCE. Anestésicos Locais: Como Escolher e Previnir Complicações Sistémicas. Rev Port Estomatol Med Dent Cir Maxilofac. 2010; 51(2):113-20.
14. Vanconcellos RJH, Antunes AA, Medeiros MF, Genu PR. Conhecimento dos alunos de graduação da FOP/UPE em relação à indicação de anestésicos locais para pacientes especiais. Odonto. 2010; 18(35): 30-6.

15. Guzmán Alvarez R, Campos Sepúlveda AE, Matínez González AA. Knowledge about local anesthetics in odontology students. Proc West Pharmacol Soc. 2009; 52: 118-9.

16. Veering BT. Complications and local anaesthetic toxicity in regional anaesthesia. Curr Opin Anaesthesiol. 2003; 16(5): 455-9.

17. Ranali J, Andrade ED, Volpato MC. Profilaxia, tratamento e controle do paciente com doença sistêmica ou que requer cuidados especiais. In: Todescan FF, Bottino MA. Atualização na clínica odontológica. São Paulo: Artes Médicas; 1996. 765-89.

18. Antunes AA, Vanconcellos RJH, Medeiros MF, Genu PR. Conhecimento dos alunos de graduação da FOP/UPE em relação à dosagem anestésica local. Rev Cir Traumatol Buco-Maxilo-fac. 2007; 7(1): 71-78.

19. Carvalho B, Fritzen EL, Parodes AG, Santos RB, Gedoz L. O emprego dos anestésicos locais em Odontologia: Revisão de Literatura. Rev Bras Odontol. 2013; 70(2): 178-81.

Submetido em: 12-09-2016

Aceito em: 20-06-2017 